\title{
Challenges and opportunities in clinical translation of biomedical optical spectroscopy and imaging
}

\author{
Brian C. Wilson \\ Michael Jermyn \\ Frederic Leblond
}




\title{
Challenges and opportunities in clinical translation of biomedical optical spectroscopy and imaging
}

\author{
Brian C. Wilson, ${ }^{\mathrm{a}}$ Michael Jermyn, ${ }^{\mathrm{b}}$ and Frederic Leblond ${ }^{\mathrm{c}, \mathrm{d}, *}$ \\ anniversity of Toronto and Princess Margaret Cancer Centre/University Health Network, Department of Medical Biophysics, \\ Toronto, Ontario, Canada \\ ${ }^{b}$ Dartmouth College, Thayer School of Engineering, Hanover, New Hampshire, United States \\ 'Polytechnique Montreal, Department of Engineering Physics, Montreal, Québec, Canada \\ dCentre de Recherche du Centre Hospitalier de I'Université de Montréal, Montreal, Québec, Canada
}

\begin{abstract}
Medical devices face many hurdles before they enter routine clinical practice to address unmet clinical needs. This is also the case for biomedical optical spectroscopy and imaging systems that are used here to illustrate the opportunities and challenges involved. Following initial concept, stages in clinical translation include instrument development, preclinical testing, clinical prototyping, clinical trials, prototype-to-product conversion, regulatory approval, commercialization, and finally clinical adoption and dissemination, all in the face of potentially competing technologies. Optical technologies face additional challenges from their being extremely diverse, often targeting entirely different diseases and having orders-of-magnitude differences in resolution and tissue penetration. However, these technologies can potentially address a wide variety of unmet clinical needs since they provide rich intrinsic biochemical and structural information, have high sensitivity and specificity for disease detection and localization, and are practical, safe (minimally invasive, nonionizing), and relatively affordable. () 2018 Society of Photo-Optical Instrumentation Engineers (SPIE) [DOI: 10.1117/1.JBO.23.3.030901]
\end{abstract}

Keywords: optical spectroscopy; optical imaging; clinical translation.

Paper 170646VR received Oct. 4, 2017; accepted for publication Jan. 24, 2018; published online Mar. 6, 2018.

\section{Introduction}

As the enabling technologies for biomedical optical spectroscopy and imaging (OSI) continue to proliferate, so also do the range of existing and potential applications: in the life sciences, tools to study the structure and functions of molecules, cells, and organisms; in forensic science to study biomolecules at a crime scene; in biotechnology and pharmaceutical industries, tools for R\&D and product quality control; and in clinical medicine, tools for screening, diagnosis, interventional guidance, treatment response monitoring and treatment of disease. OSI is, of course, a subset of the larger biophotonics landscape that includes medical diagnostics and therapeutics as well as tools for biomedical research. ${ }^{1}$ There is increasing recognition of the need to debate the opportunities and challenges in bringing biomedical technologies, including light-based approaches, from the concept stage through preclinical studies, clinical trials, commercialization, and into routine clinical adoption. ${ }^{1-3}$ The focus of this paper is to consider the particular challenges and potential solutions for the translation of OSI into the clinic, either ex vivo (biofluids including blood, urine and saliva; tissue biopsies; cells) or in vivo (non- or minimally invasive probing in live patients). It will become clear that most biotechnologies face similar challenges in clinical translation, so that much of this discussion is equally applicable to nonoptical approaches. ${ }^{2}$ However, wherever possible we will use examples drawn from OSI to illustrate the issues and will also indicate

*Address all correspondence to: Frederic Leblond, E-mail: frederic.leblond@ polymtl.ca throughout what challenges and opportunities are specific to these optical technologies.

OSI technologies can be used at one or more stages for clinical management (Table 1): to diagnose or localize disease, ${ }^{4,5}$ to guide interventions, ${ }^{6-8}$ or to monitor treatment response and safety and disease progression..$^{9,10}$

OSI techniques have been used for cancer screening using measurements of tissue or biofluids, ${ }^{11,12}$ integrated into clinical biopsy tools, ${ }^{13}$ and for disease (especially tumor) margin detection and localization. ${ }^{14,15}$ It should be noted that treatment itself may also be optics-enabled, using light as an energy source to modify or destroy cells/tissue through photochemical, photothermal, or photomechanical interactions. ${ }^{11,16,17}$ These treatment modalities have been one of the major drivers for the development of OSI: for example, the development of in vivo fluorescence spectroscopy imaging to detect the presence and distribution of photosensitizer molecules during photodynamic therapy (PDT) has subsequently been transferred into a broader range of applications using multiple optical contrast agents and technical approaches. ${ }^{17}$ OSI may be used also as part of the clinical toolkit in nonlight-based therapies, including surgery, chemotherapy, and radiation therapy. For example, 5-aminolevulinic acid (5-ALA) fluorescence-guided neurosurgeries facilitate intraoperative visualization of gliomas, allowing surgeons to achieve maximal resection of tumors, resulting in improved survival and quality of life. 7,18,19

Rather than providing a general review of OSI technologies in clinical applications, we will discuss primarily the issues and challenges that arise in moving these techniques from the concept and laboratory-prototype stages through commercialization to adoption and dissemination into routine clinical practice. This 
Table 1 Applications for optical technologies at different clinical stages.

\begin{tabular}{|c|c|c|}
\hline Screening and diagnosis & Treatment & $\begin{array}{l}\text { Monitoring treatment response } \\
\text { and disease progression }\end{array}$ \\
\hline $\begin{array}{l}\text { Screening for early disease detection } \\
\text { using optical spectroscopy of tissue or } \\
\text { biofluids }\end{array}$ & $\begin{array}{l}\text { In situ disease stratification to inform } \\
\text { treatment decision-making }\end{array}$ & $\begin{array}{l}\text { Prognostication to better } \\
\text { stratify disease and provide } \\
\text { tailored treatment }\end{array}$ \\
\hline $\begin{array}{l}\text { Integrating optical techniques into } \\
\text { clinical biopsy tools to avoid } \\
\text { complications and provide diagnostic } \\
\text { predictors }\end{array}$ & $\begin{array}{l}\text { Guidance for surgical resection } \\
\text { - margin assessment/localization } \\
\text { - local drug administration } \\
\text { - focal therapy (e.g., brachytherapy) }\end{array}$ & $\begin{array}{l}\text { Monitor disease progression } \\
\text { assess outcome, flag residual } \\
\text { disease }\end{array}$ \\
\hline $\begin{array}{l}\text { Complementing current standard-of- } \\
\text { care diagnostic procedures by } \\
\text { providing additional information for } \\
\text { combined analysis techniques }\end{array}$ & $\begin{array}{l}\text { Optics-enabled treatment using } \\
\text { photochemical, photothermal, or } \\
\text { photomechanical interactions to treat disease }\end{array}$ & $\begin{array}{l}\text { Monitor treatment safety, } \\
\text { especially for chronic conditions } \\
\text { and long-term treatments }\end{array}$ \\
\hline
\end{tabular}

technological translation includes what is commonly referred to as the "bench-to-bedside" paradigm but goes beyond it to consider the full spectrum of challenges: scientific/technological, biological, clinical, commercial/industrial, regulatory, socioeconomical, geographical, and cultural. We will see, however, that some of these (apparent) challenges also represent significant opportunities. Hence, despite the fact that OSI technologies face considerable hurdles in being fully translated, there are many opportunities to exploit the unique characteristics of optical techniques, particularly at the molecular level, which contrast with other medical imaging methods, such as X-ray-based techniques that interact primarily at the atomic length scale.

The hope is that this paper will provide guidance to help accelerate the translation. To this end, we will first discuss the fact that commercialization is a critical part of clinical translation. The choice between "technology push" and "market pull" will then be discussed as alternative routes to clinical translation. After considering some of the barriers to clinical adoption of new technologies, the specific steps in clinical translation will be considered in more detail, following which issues in technology prototyping and competitive performance will be addressed. We will end by identifying other, perhaps unexpected, challenges faced in clinical translation. Broadly speaking, the fate of an optical technology for clinical adoption depends upon how useful it is in a clinical setting (information content, minimal disruption, etc.) and the scientific/technological maturity of the technique. The technology must address an unmet clinical need in a practical manner, which is facilitated by technological advances and impacts when and how the clinical requirements can be met.

\section{What Are Biomedical Optical Spectroscopy and Imaging Technologies?}

Tissues are comprised of cells and intercellular structures/fluids with particular structural characteristics as well as dynamic biomolecular composition. Diseased cells/tissue may exhibit significant morphological, molecular, mechanical, and/or and biochemical changes as compared with normal cells/tissue. ${ }^{20}$ The corresponding optical properties can be valuable for clinical utilization because of their unique characteristics revealing morphological, molecular, and biochemical tissue signatures. ${ }^{21}$
In the present context, we define optical spectroscopy as the measurement of the optical properties of a biospecimen (biofluid/cells/tissue/patient), i.e., measurements following light interactions with the specimens. These interactions include absorption, elastic scattering, fluorescence, and inelastic scattering (Raman), all of which are linear in nature, i.e., the interaction probability is proportional to the optical power (density) applied to the sample. In addition, several nonlinear processes are rapidly emerging in OSI, including multiphoton fluorescence, ${ }^{22}$ second- and third-harmonic generation, ${ }^{23,24}$ coherent antistokes Raman scattering, ${ }^{25}$ and stimulated Raman scattering. ${ }^{26}$ Additional optical phenomena may be used in combination with some of these interactions, such as interference [e.g., optical coherence tomography (OCT)] and polarization. ${ }^{27,28}$ Probing multiple wavelengths in optical imaging provides increased biomolecular information, which often confers added molecular specificity. This is critical for many imaging techniques such as Raman spectroscopy, in which much of the diagnostically relevant information cannot be captured in a single spectral feature. ${ }^{6,29-31}$ Optical spectroscopy techniques can have vastly different spatial resolutions (from microns to millimeters), penetration depths, and target sensitivity/specificity. Choosing an appropriate technique for a particular clinical application must take many such factors into consideration, which can affect diagnostic and imaging performance, instrument size, ease-ofuse, cost, and impact on the clinical workflow.

The general advantages and limitations of optics-based technologies are summarized in Table 2: these include intrinsic characteristics that are fundamental to the nature of the interactions between light and the biospecimen, as well as practical considerations that can impact clinical use.

Optical techniques are varied, and so OSI approaches are usually designed and tailored for a specific clinical application: from the technical and clinical specifications of the system to the availability and cost of optical components. This is very different from established modalities such as those used, for example, in radiological imaging, where the instruments ( $\mathrm{x}-$ ray, CT, MRI, PET) are "monolithic," i.e., large, expensive, and centralized machines that are used across many different diseases and applications. This presents OSI with a different set of challenges and opportunities for clinical translation and commercialization. 
Table 2 Fundamental ("intrinsic") as well as practical advantages and limitations specific to OSI in clinical medicine.

\section{Fundamental}

$\begin{array}{ll}\text { Advantages } & \text { Molecular sensitivity } \\ \text { Multiple interaction mechanisms providing different } \\ \text { information (e.g., morphology, metabolism) or } \\ \text { therapeutic effects } \\ \text { Spectral multiplexing } \\ \text { High "target" specificity } \\ \text { Many options in photoactive compounds for } \\ \text { diagnostic contrast or phototherapeutic targeting } \\ \text { Very large range of size scales: from single } \\ \text { molecules to whole organs } \\ \text { Nonionizing } \\ \text { Relatively low penetration of light in tissues } \\ \text { Strong trade-off between spatial resolution and } \\ \text { depth sensitivity due to strong light scattering } \\ \text { Complexity of the optical signals } \\ \text { Minimal detectable concentration of biomarkers } \\ \text { limited by signal to background ratio }\end{array}$

\section{Practical}

Advantages $\begin{aligned} & \text { High level of safety: noninvasive or minimally } \\ & \text { invasive }\end{aligned}$

Compact instrumentation

Relatively low cost

Applications across many clinical specialties

Ease of integration leading to multimodal systems

Compatibility with other modalities

Long-term stability and robustness of optical components

Limitations Systems often application-specific: few "generic" solutions/technologies

Sensitive to ambient and external sources of light

\section{Versatility of Optical Systems to Address Clinical Needs}

Point spectroscopy, i.e., measurement at a single location on the biospecimen, may be implemented using an open-beam optical geometry, as commonly used for ex vivo analysis, or via optical fibers that enable remote spectroscopy in vivo, including deep in the body via endoscopes or during open surgery (so-called "optical biopsy"). ${ }^{32}$ Spectral imaging here refers to mapping of the spatial distribution of the optical interactions across the sample. This may be achieved either by physically scanning the sample (in the ex vivo case), by scanning an optical fiber probe, or by illuminating the sample across a wide field of view and using a detector array with appropriate light-collection optics. ${ }^{33}$ There is a fundamental technological trade-off in spectral and spatial information content between these two modalities, point versus imaging. Thus, point spectroscopy can be performed with high spectral resolution across a wide spectral range, whereas spectral imaging usually involves using only a few wavelengths or wavelength bands to achieve cost-effective performance in a clinically acceptable time. This translates into substantive compromises in clinical applications, as will be discussed as follows. Notably, optical techniques probe different length scales depending on the system and application: microscopic $(<0.1 \mathrm{~mm})$, macroscopic $(>1 \mathrm{~cm})$, or mesoscopic (between microscopic and macroscopic). Single-point and wide-field imaging techniques target different scales and, therefore, different biological attributes.

A limited number of OSI technologies have reached routine clinical practice at this time. Some of these are well established, such as white-light endoscopy ${ }^{34,35}$ and white-light and fluorescence imaging in the eye ${ }^{36}$ while others such as OCT has been adopted more recently ${ }^{37}$ but do not fully utilize spectral information at this time. Spectrally resolved diffuse reflectance or transmittance spectroscopies are used routinely for pulse oximetry and brain oxygen monitoring in neonatology. ${ }^{38}$ To place this in perspective, while only a handful of other imaging technologies (CT, ultrasound, radionuclide/PET, and MRI) have been translated into routine clinical practice since the discovery of $\mathrm{x}$-rays in 1895 , each has evolved almost unrecognizably in technological performance and has achieved high penetration across a range of clinical applications. Nevertheless, many clinical applications remain where these established modalities are inapplicable or do not provide adequate biomedical information. It is also the case that some individual OSI technologies have undergone huge technical advances. For example, enabled by advances in component hardware and software, optical coherence tomography has increased in imaging speed by some 500,000 -fold and up to 1000 -fold in sensitivity over the past 25 years during which this technique has gone from a lab tool to a routine clinical modality for retinal scanning with $>30$ million scans performed worldwide per year. Likewise, optical imaging of hollow organs has progressed from visual inspection through simple rigid tubes to multimodality flexible endoscopes operating in real time and even imaging within a single artery. ${ }^{39}$ The "nimbleness" of optical technologies is a distinct advantage in this regard.

\section{Commercialization as a sine qua non for Clinical Translation}

With few exceptions (e.g., some point-of-care technologies developed and disseminated through global philanthropy), having a technology reach and impact patients requires that it be commercialized, i.e., becomes a "product." In general, commercializing clinical technologies is more complex, takes longer, and is more expensive than for nonclinical (i.e., consumer) products. The typical development pathway for consumer products involves moving from the idea through initial scientific investigation and validation, building of the business case, prototype development and testing, product engineering design, manufacturing, marketing and postsales support. All these steps are also required for clinical technologies. However, there are essential additional tasks, in particular, clinical trials to determine safety and efficacy, obtaining regulatory approvals, which incur increased difficulty and larger resources, negotiating 
Table 3 Pros and cons of alternative developmental pathways to clinical translation.

Technology push

Advantages Retains maximum flexibility

Builds on already-developed technologies

Limitations May not find viable clinical applications

Need to identify and persuade clinical development partners

Hard to get investors without clear "clinical customer" endpoint

Hard to get translational grants for the R\&D phases

May need significant redesign once a specific clinical application is identified

End-user physicians generally do not care whether or not the technology uses optics

Clinical pull

Advantages

Easier to "sell"

Clinical champions from day 1

Higher chance of clinical translation

Limitations

May miss an even more important application

The presence of a need does not mean that a technology solution will be found

reimbursement for use, and postuse safety monitoring and in some cases also efficacy studies to extend the indications.

Clinical trials include several distinct stages: phase I (initial safety and technical feasibility), phase II (demonstration of efficacy, i.e., that the technology does what is intended) and phase III (demonstration that the technology is at least equivalent to existing approved alternatives). These clinical trials often represent a large fraction of the time and money required for clinical product development. In addition, there are usually one or more substantive iterative loops in the process that further increase these costs, e.g., findings made during the clinical trial may force redesign of the instrument or even additional preclinical studies in cells or animal models. The motivation to go through these procedures is, of course, the expectation of large revenues and/or high clinical impact once the technology is adopted into widespread clinical use.

A particular challenge with commercializing OSI technologies springs from the fact that there are often multiple possible technical approaches and many different clinical applications, each combination of which represents one potential commercial product that may have a limited and specialized market. For example, the use of fiberoptic Raman spectroscopy has recently been reported to guide brain tumor surgery, ${ }^{6,40}$ with highly encouraging results in terms of sensitivity and specificity, validating the scientific and technical approach. Several questions then arise. Is the market large enough for the product to be commercially viable? Can the same system be used for other clinical applications and, if so, would this require specific clinical trials and/or major technical modifications (e.g., for endoscopic use)? Can the device be based on a common hardware and software platform across multiple applications or, for example, would different spectral analysis algorithms be needed for each application? Would the system require regulatory approval for each use or could there be a more generic approval? How could a company access a diverse range of clinical specialists and markets, each with their own particularities? These are substantial but exciting challenges.

\section{Route to Clinical Translation}

\subsection{Alternative Pathways to Translation}

There are two distinct pathways that can be followed in developing clinical technologies: technology push or clinical pull. The first often includes technologies that were developed initially for nonclinical purposes and then possible clinical applications are sought to expand the market. In the second approach, the technology is conceived and developed in response to a clearly-defined existing unmet clinical need. Table 3 highlights a few of the advantages and risks/challenges of each approach.

In a recent paper by Beswick et al., ${ }^{2}$ it is stated that "... only once the clinical need be understood in detail can the invention process begin," but this ignores the fact that OSI in particular has often been driven by new and disruptive technologies from the broad photonics sector as well as by advances in molecular sciences/chemistry (e.g., biomarker-targeted, optically-active "reporters") and materials science (e.g., photonic nanotechnologies for use in imaging or treatment): i.e., the technology came first and opened up possibilities for addressing the clinical needs. In any case, the unmet need is most likely identified first by a physician who then seeks out research scientists and technologists and/or companies to develop OSI technologies to address the need. Knowing exactly (or even roughly) the intended final clinical use and having strong guidance from the "end-users" are important, especially since "inventors" are not necessarily skilled and experienced in clinical-needs assessment. Productive interactions between scientists/engineers and clinician require significant effort to break down the interdisciplinary knowledge and language barriers, especially if the former are based in a purely academic environment rather than, say in a medical research institute that is part of an academic hospital where there are many more opportunities for ongoing interactions: Popp et $\mathrm{al}^{3}{ }^{3}$ have also discussed the benefits of multidisciplinary research centers established specifically for developing and translating (photonics-based) technologies and give examples of such initiatives and how they might be funded. Regardless of the translational model, in most cases it is the physicians rather than the patients who determine whether a technology will be adopted, but considering the patient perspective is also valuable, preferably at an early point in the translation process.

A good example of clinical pull for OSI technologies is the use of in vivo Raman spectroscopy for surgical guidance of (brain) cancers. ${ }^{6,40}$ The unmet clinical need for rapid and accurate tumor tissue detection and localization during surgery was identified by clinical users and the OSI technology, based on point Raman spectroscopy, was then developed and tailored to address this specific need [Fig. 1(a)] and a company was formed to commercialize the technology (ODS Medical Inc., 

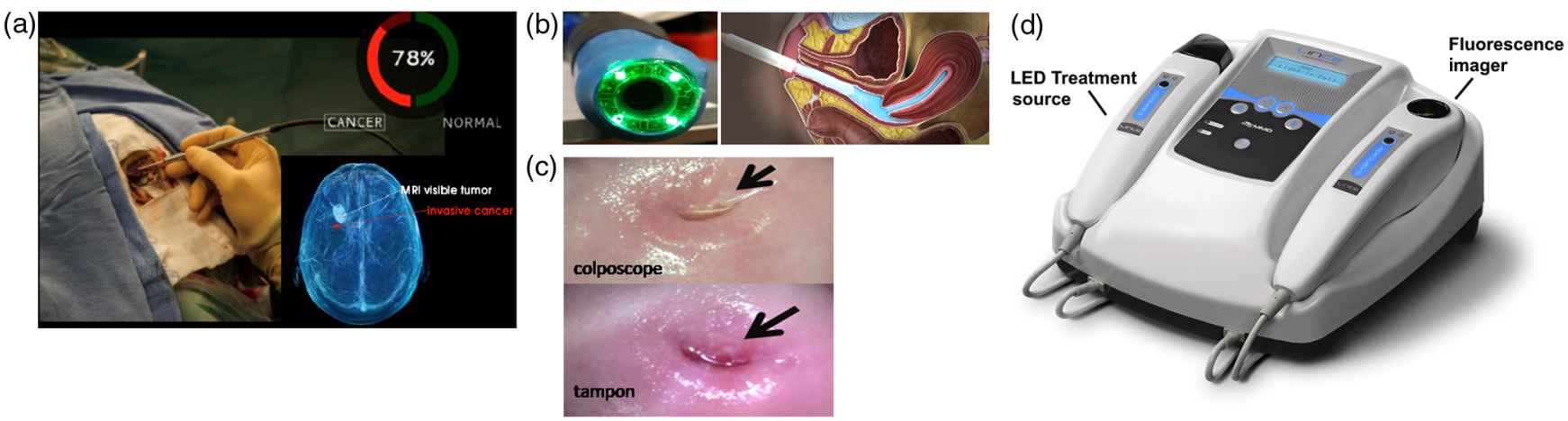

Fig. 1 (a) Raman spectroscopy system for surgical guidance of brain cancers, illustrating the use of a Raman spectroscopy probe in situ to distinguish normal brain from cancer (courtesy of ODS Medical Inc.). The bottom right shows a volumetric rendering of the MRI-visible tumor, relative to invasive cancer that extends into the normal brain. (b) Diagnosis of cervical cancer using optical imaging with conventional colposcope (left) and tampon-like device (right). (c) Comparison of image quality between the two technologies in B (B and C courtesy of Dr. Nirmala Ramanujam, Duke University). (d) Device utilizing fluorescence imaging and PDT for skin cancer (D courtesy of MM Optics, Brazil).

Canada) [Fig. 1(a)]. The use of clinical pull can also facilitate the running of clinical trials through having preexisting collaborative effort and motivation on the clinical side. Examples of successful technology pushes also exist and include technology, which was initially developed without a specific clinical objective in mind but was nevertheless able-through rapid technological advances and productive relationships developed between the technical and medical sectors-to find specific clinical application. OCT is a good example of this, where important clinical applications were identified such as in ophthalmology and gastroenterology. Several companies were created that are now manufacturing OCT clinical systems.

\subsection{Barriers to Clinical Adoption}

With consumer technologies, whether they be cell phones or automobiles, the primary determinants of adoption (apart from luck and marketing) are "Do I need or want this?," "Can I afford it?," and "Is this the best model?" In considering the barriers to a new clinical technology being widely adopted, it is worth listing the multiple factors involved, some of which are common to consumer products while others are distinctly different and involve a much more diverse set of stakeholders, namely inventors, academic institutions and medical centers, investors, physicians, patients, health-care providers, and governments that need to be engaged at different stages. ${ }^{2}$ The barriers include: scientific and technical, clinical, socio-economic, geographic, cultural and educational, as can be illustrated by a recent and striking example. Cervical cancer is a significant health-care problem worldwide but in particular, in the words of the World Health Organization, it is "an avoidable cause of death in sub-Saharan Africa," where the incidence is five times higher than in the USA and the death rate is nine times higher. An existing optical technology, colposcopy [Figs. 1(b) and 1(c)], is used in developed countries to diagnose cervical cancer at a treatable stage, in which the surface of the cervix is viewed through an optical probe placed close to the cervix to identify areas of abnormality. Hence, the scientific and technical barriers have already been overcome. What then are the barriers to adoption to address the clear unmet clinical need in sub-Saharan Africa and other parts of the developing world? The first is a combination of clinical and educational factors; there are not enough trained gynecological oncologists to meet the workload. The second is socioeconomic, since a colposcope costs around USD $\$ 20,000$, which is beyond the means of many local healthcare systems. Geographic barriers then come into play because of the difficulty in providing high-tech medical services at the "village"-level across large areas. Another major barrier is cultural, in that there is fear and reluctance on the part of patients and their families to have this examination, especially if performed by a male doctor. Recently, a radical alternative has been developed [Figs. 1(b) and 1(c)], in which the optical system has been markedly reduced in size and cost and is in the form of a tampon that it inserted by the woman herself. ${ }^{41}$ The digital images may then be sent via cell phone to a central expert reader for diagnosis. Histological images of tissue samples taken under this image guidance could also then be generated locally using a cell phone-based microscope and transmitted to a central expert pathologist. This is a compelling example of an optical technology being developed/adapted for a defined unmet clinical need rather than vice versa. Clearly, the approach is relevant to a wide range of possible clinical needs and OSI solutions.

Another example of designing optical technologies to meet clinical needs at locally affordable cost, and that are practical for dissemination across large countries with geographically variable medical infrastructure, is in PDT, i.e., the use of compounds that are activated by light to kill abnormal cells or organisms. ${ }^{16,17}$ A good example is a group at the University of Saõ Paulo in Brazil that has re-engineered a LED-based light source for PDT treatment, ${ }^{42}$ used in combination with a simple fluorescence imager to delineate the tumor and guide treatment, into a low-cost, reliable, user-friendly package about the size and format of a desk telephone [Fig. 1(d)]. This has been disseminated, with appropriate training, to about 80 local clinics in Brazil and the program has spread to other countries in South and Central America. Both examples demonstrate that overcoming these barriers also provide new opportunities for research, development and commercialization.

\section{Steps in Clinical Translation}

The steps toward clinical translation are shown in Fig. 2. Some of the steps can be reordered or carried out in parallel to reduce costs and accelerate the process. 


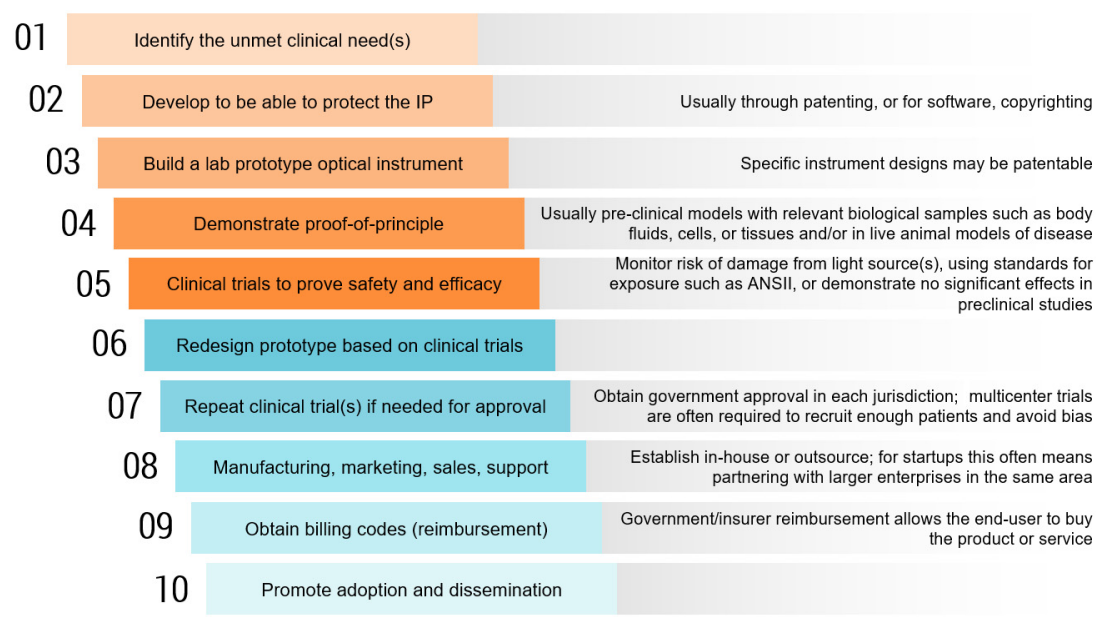

Fig. 2 Steps toward clinical translation of optical technologies.

\subsection{Preclinical Phases}

The technology development and intellectual property (IP) protection leading up to the demonstration of proof-of-principle is usually carried out in preclinical models with relevant biological specimens, and these data are often used to support patenting. Issues relating to preclinical animal models are discussed in more detail in Sec. 9.2 as follows. Although the preclinical steps in translation can be completed relatively quickly and without large capital expenditure for some simpler OSI technologies, ${ }^{2}$ this is not usually the case for "high-end" technologies that require building complex prototypes in the case of medical devices (e.g., surgical robots or multimodal imagers) and/or optically active imaging of therapeutic agents, where the synthesis and testing costs may be very high.

\subsection{Clinical Trials}

First clinical trials may include demonstrating that performing the OSI procedure on ex vivo human tissue samples (e.g., biopsies) does not compromise subsequent clinical histopathological diagnosis. Clinical trials require explicit institutional approvals with informed patient/subject consent and, depending on the level of potential risk (and, for optical systems, the laser classification), may also require government regulatory approval. Prototypes will likely be redesigned based on the results of the clinical trials. Optimal synchronization of the clinical trials with the transition from prototype to product is a critical challenge that has significant cost implications. Often, multicenter trials are required for final approval, both to recruit enough patients in a reasonable time and to mitigate against user bias. As well as demonstrating safety, the efficacy of the technology compared with other "predicate" technologies, if they exist, must be demonstrated with statistical validity. The number of patients and/or samples required in these trials can be large: for OSI, late-phase trials typically involve many tens or even hundreds of patients, and there is increasing recognition that this number is often seriously underestimated. ${ }^{43,44}$ The number of patients or samples/measurements required depends on the particular technology, what biological characteristics are being measured, the magnitude of expected effects, and the clinical endpoints of the trial. A major factor that drives up the patient numbers is the inter- and intrapatient variability. Given that one is usually investigating a disease, it might be expected that the biochemical or structural changes could vary markedly within the clinical trial population and depend also on the stage of disease. The nondisease background signals, both between patients and within an individual, can also be very high and variable and the impact of this must be minimized within the clinical trial design. Additionally, data processing (calibration, normalization, choice of classification model) can help correct for these variations. For example, the variability between spectra of the "normal" host tissue taken in different patients or even at different locations in the same patient can mask the differences due to disease, so an effective strategy is to normalize the spectra from suspected tumor to that taken in the adjacent normal tissue in the same patient. The requirement to have large numbers of samples is also generally more severe in "label-free" OSI techniques than when using an exogenous contrast agent. This is especially the case when the biological information is distributed across the optical spectrum, as for example, in Raman spectroscopy. It is less of a problem if there is a distinct spectral feature to differentiate disease from normal, as for example, the red-to-green ratio that has been used in some autofluorescence systems. ${ }^{45-47}$ For regulatory approval of a commercial product, the so-called registration trials upon which approval is granted must use a device that is substantially equivalent to that commercial product. Note also that the requirements for approval of "devices" are different from those for "drugs." Many OSI technologies are deviceonly based but if, for example, an exogenous fluorescence contrast agent or photoactive agent is also used, then drug-device combination will require approval, even if the compound is already approved for another purpose.

\subsection{Failure in Clinical Adoption}

Unfortunately, there are examples of OSI technologies that have been taken all the way through approvals only to find that there is no market. The possible reasons for this failure of adoption are several:

- The performance does not (fully) address the intended unmet clinical need. This should become clear during clinical trials, but it is a common mistake to think that "statistically significant" is the same as "clinically significant," e.g., proving in a clinical trial that the OSI 
procedure improves the detection of tumors by $10 \%$ (with a small $95 \%$ confidence interval: i.e., statistically sound) does not mean that the clinical users or the health-care system (payers) will think that this incremental benefit is worth the cost and effort involved.

- The technology is too expensive. OSI has a distinct advantage in this respect, since most of the enabling components (light sources, photodetectors, etc.) come from the much larger consumer photonics sector, where sales volume drives down the unit price over time, often very substantially: for example, the cost per Watt of LEDs has plummeted in the last decade, while compact monolithic spectrometers are becoming available with acceptable performance for some clinical applications. Hence, an OSI system that is too expensive today may well be competitive in a few years and can be worthwhile to revisit.

- There is competitive technology already on the market for the same clinical application. A good example of this is in the identification of positive margins in breast cancer lumpectomy. This is an important clinical need to reduce the number of second surgeries performed as a result of tumor being found extending to the surface of the surgical specimen but not being discovered until days or weeks later when the traditional histopathology report becomes available. Several OSI methods are being explored for this application, including fluorescence spectroscopy/imaging with and without exogenous contrast, ${ }^{48-50}$ optical coherence tomography, ${ }^{14}$ and point Raman spectroscopy. ${ }^{15} \mathrm{~A}$ variety of nonoptical techniques are also under development. For example, a radiofrequency device ${ }^{51}$ has already received FDA approval and is commercialized, which becomes a likely predicate device against which any new OSI device will be evaluated: note, this does not necessarily mean that a head-to-head clinical trial is required, but rather that the clinical performance of the OSI system (e.g., sensitivity and specificity for detecting positive margins) needs to be at least equivalent in terms of the intended use. Developing a clear regulatory strategy early in the translation process can help minimize the costs of obtaining governmental approval, as has been discussed by Beswick et al. ${ }^{2}$

- The technology is too "clunky," i.e., has poor ergonomics for use in the intended clinical environment, or has a poor user interface (e.g., complex, confusing, or delayed display of the data), or requires too much technical support in the clinic.

- The clinical setting is not ready for the technology. An example is autofluorescence endoscopy, based on specific spectroscopic differences between normal bronchial tissues and early cancer, ${ }^{4,52}$ where a system was developed commercially in the 1990s and received FDA approval for detection of pre- and early-stage lung cancer but was never widely adopted. This was not because of inadequate technical or clinical performance but because no effective treatment could be offered to the patients, so that there was little motivation for physicians (primarily, respirologists) to adopt the technique. However, the same technology was subsequently bundled with white-light and narrow- band endoscopy (multispectral imaging) and adopted into gastrointestinal endoscopy, where minimally invasive endoscopic treatments are already in place. This is a good example of "repurposing" of an OSI technology to address a different unmet clinical need.

\section{Prototypes Are Not Products}

Technologies invariably go through several prototypes before settling on the design and manufacture of the final commercial and approved product. Many OSI researchers have neither the time nor the resources to bring early-stage prototypes through to a product ${ }^{3}$ and there are often substantial differences between these. This is particularly true for optical systems, where the complexities of prototypes are often not feasible for production. Figure 3(a) shows a classical OSI prototype that was developed for fluorescence guidance in brain tumor surgery using exogenous fluorophore contrast. ${ }^{53}$ The system comprised of a sophisticated "point and shoot" white-light and fluorescence camera, integrated with a joystick-controlled remote point spectroscopy unit. This was demonstrated successfully in clinical trials. However, subsequently it was considered "unmanufacturable," i.e., it could not be reproduced in volume at acceptable cost. The reason was intrinsic to the optical design, wherein the fluorescence excitation optical path was coaxial with the fluorescence detection path: since the fluorescence coming back to the camera is several orders-of-magnitude weaker than that the backscattered excitation light from the tissue, specular reflections at all optical surfaces caused unacceptable background in the fluorescence images even after spectral filtering. Hence, all the optical elements needed to be slightly angled to the optical axis, which was impractical in a manufactured product. The system was totally redesigned, as shown in Fig. 3(b), by separating the excitation and detection paths, but with reduced functionality, including eliminating the spectroscopic feature. However, this process of first building a "Rolls Royce" prototype can still be valuable, especially if the underlying science is not fully mature, since the information gained from a system with maximum functionality can provide critical information to design the more translatable system.

The second example, also in the domain of fluorescence imaging, is shown in [Figs. 3(c) and 3(d)], comprising a cell phonebased device. The first commercialized clinical application of the device is to localize, through imaging of their autofluorescence, bacteria in the region of chronically infected skin wounds (e.g., diabetic ulcers), enabling the wounds to be properly cleaned to accelerate healing. ${ }^{54}$ The prototype in Fig. 3(c) clearly has several suboptimal features: it is not easily cleaned or sterilized, is not very robust (in particular, the LEDs are exposed and can be damaged easily), has poor aesthetics, would be difficult to manufacture reliably in volume, and is not particularly user-friendly. Nevertheless, this prototype was employed successfully in clinical trials that provided enough data to enable it to be redesigned/re-engineered into a commercial product [Fig. 3(d)]. This final product probably could not have been produced in a typical research laboratory environment, but the clinical functionality of the prototype and product was close, so that the clinical trial data from the former were considered valid for regulatory approval.

Other examples include technologies that were initially developed for specific clinical applications but ended up being successfully translated for other applications. For example, the initial use of fiber optic-based point Raman 
(a)

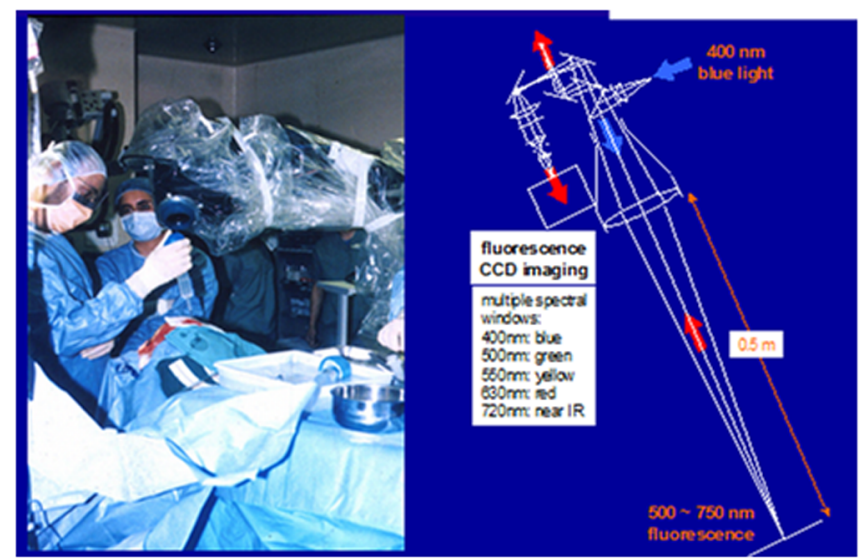

(b)

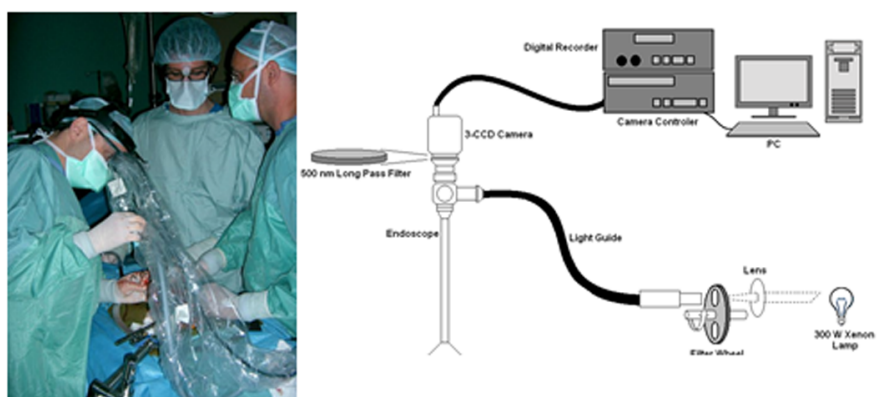

(c)
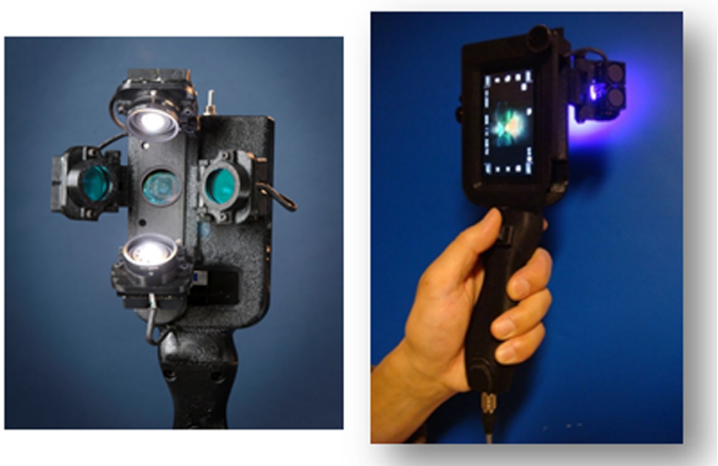

(d)

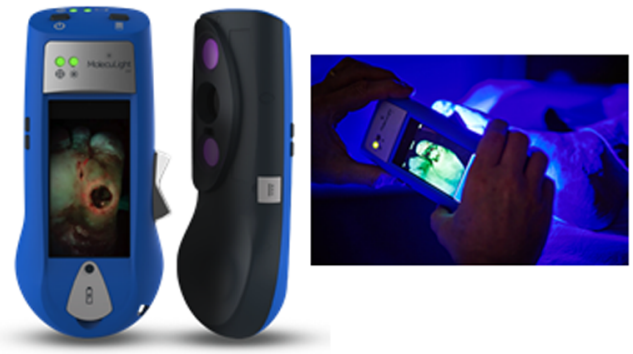

Fig. 3 Examples comparing "prototypes" and "products." (a) First version of fluorescence imager with point spectroscopy (left: in use to guide brain tumor surgery, right: optical schematic), (b) after reengineering into a compact multispectral "ratiometric" imaging device, but excluding point spectroscopy (left: in use during prostate cancer surgery, right-optical schematic), (c) first prototype of cell phone camerabased fluorescence imager used in clinical trials, (d) re-engineered commercial version (courtesy of Moleculight Inc. Toronto. Canada).

spectroscopy in the clinic was for endoscopic applications. While the clinical results were generally positive for a number of pathologies, e.g., in the GI tract ${ }^{30}$ limited commercial interest impeded progress and prevented clinical translation and commercialization. In part, this was due to the fact it was not clear how the newly provided biological information would actually affect clinical practice since endoscopists would probably still collect biopsy samples for medical-legal reasons regardless of the information provided by the OSI technology. Moreover, the actual need in endoscopy was for a complementary fast wide-field imaging technique with good sensitivity to guide the Raman spectroscopy sampling, a functionality that was not provided by single-point fiber-optics solution. However, technological advances in lasers and photodetection approaches, as well as in key enabling filtered fiber technology, led to the revival of point-based Raman spectroscopy for different applications, including surgical guidance and targeted biopsy guidance in brain surgery. ${ }^{30}$ These advances surmounted the critical clinical translation barriers and the technology is in current company-sponsored clinical trials.

\section{Trade-Offs in Performance}

In developing clinical OSI technologies, there are inevitable compromises between technical performance, cost, and clinical utility. The performance of the final product in the intended clinical setting must substantively address the clinical need, sustainably, and profitably against competing (nonoptical) solutions. It is worth breaking down this challenge into its major elements.

\subsection{Technical Factors}

With regard to the scientific and technical trade-offs, the first concerns the quality of the information obtained in the clinical procedure. In the case of optical spectroscopy, this includes the signal-to-noise and signal-to-background ratios and the spectral and spatial resolutions. These need to be balanced against the time required to collect spectra or spectral images. In turn, these technical performance factors are likely to be reflected in the cost of the instrument, for example, the quality (sensitivity, linearity, dynamical range of detection, speed, etc.) of the spectrometers and photodetectors used, as well as their size and complexity. In the second trade-off, the main issue around quantity of information is the sampling density of the measurements, i.e., the number of discrete points on the sample/tissue at which spectra are collected, or the field-of-view and pixel density in the case of spectral images. This often has critical clinical implications, for example, to minimize the risk of missing diseased regions due to under-sampling. If the spatial resolution and/or imaging field of view for sampling are incompatible with the typical feature size of the disease, there is increased risk of missing disease due to partial volume sampling. The aforementioned performance factors are primarily related to the hardware in OSI systems.

\subsection{Analysis Factors}

The third factor is the choice of algorithms used in the spectral analysis software. Commonly, these are applied to the spectra or images acquired from an individual patient or biospecimen to 
classify cells or tissues as normal or abnormal (e.g., tumor). The algorithms are often chemometric in nature, i.e., the measured spectrum from the unknown specimen is used as input to a "black box" algorithm that has been trained on spectra taken from a range of prior samples in which the disease classification is known by an independent "gold standard" technique, such as histopathology or biochemistry. The optimum choice of algorithm (e.g., possibly based on principal components analysis, neural network, support vector machines, etc.) is far from trivial and can depend strongly on the nature of the data. There are also many rules and best practices in the training, validation, and implementation of the algorithms that must be followed to ensure that the resulting classification is accurate and robust. ${ }^{55,56}$ Common pitfalls include: inadequate sample numbers for training, use of the same data for training and validation, bias in the training and/or validation datasets, inadequacy of the gold standard, and using the algorithm on samples whose spectra or classification lie outside the range used in training the algorithm. ${ }^{30} \mathrm{~A}$ common trade-off is the accuracy of the algorithm versus its robustness and speed, the latter being of most concern in the case of spectral imaging where the datasets may be very large: for example, in the case of intravascular OCT imaging, the initial implementation of algorithms used to classify arterial tissues based on the textural features initially took several hours compared with a few seconds for the actual data acquisition in the patient. ${ }^{57}$ Specialized software and hardware (e.g., graphic processing units) may be required to achieve clinically useful data analysis speed, with resulting impact on the system cost. Many other areas of modern medicine involve the acquisition, analysis, management, and archiving of increasing volumes of digital data ("big data"). This is becoming an expensive challenge and the same is increasingly true for some OSI techniques, especially spectral imaging. However, the advent of more advanced machine-learning/artificial intelligence, computer-vision algorithms, and computational capability presents a significant advantage to OSI. Single wavelengths or metrics often do not capture all the clinically relevant information from multiple biomolecular or structural signatures. Faster and better machine-learning algorithms are able to rapidly identify patterns in spectral data and, thereby, provide improved spectral analysis techniques to optimize diagnostic performance. ${ }^{57-59}$ Computational power has reached the point where powerful feature extraction and classification techniques can be used, particularly for imaging data where important features in the images can be automatically identified using computer vision techniques. ${ }^{57,60}$

\subsection{Workflow and Clinical Factors}

The scientific trade-off of information content versus cost/complexity speaks to the need for making the OSI measurements fast enough to not unduly disrupt the clinical workflow. This can be particularly challenging in endoscopic and surgical procedures and it is worth determining early in the development phase what is the maximum acceptable measurement and analysis time once the technology is adopted into routine clinical use: these are usually much shorter than the time that can be tolerated by the clinical investigative team during the clinical trial phase. The target population for optimizing diagnostic performance should also be determined early, since it can significantly affect the clinical trial design and execution. For example, fluorescence-guided resection of brain tumors using the fluorophore protoporphyrin IX induced by administration of the prodrug aminolevulinic acid
(ALA-PpIX) is substantially less accurate for low grade than for high-grade gliomas, because of the much lower PpIX concentration in the former, so that the fluorescence signal falls below the limit of detection of current commercial OSI clinical instruments. On the other hand, some low-grade glioma may be curable if all tumor tissue is resected, so this represents a high impact subset of patients. One solution to address this tradeoff is to combine fluorescence and diffuse reflectance spectroscopies: the latter is used to determine the tissue absorption and scattering spectra, which are then applied to the measured fluorescence spectrum to correct for the variable attenuation of the excitation and emission light, thereby lowering the detection threshold of the fluorophore concentration in the tumor. ${ }^{61}$ This is an example where improved OSI performance extends the clinical utility and expands the potential market. It illustrates that extending the design increases the impact. This is not necessarily true in all cases and is worth determining before getting too far into clinical trials.

Balancing the size of trials against their time and cost is very critical and is usually impossible to determine a priori, since the statistical accuracy (e.g., sensitivity and specificity for disease detection) is only known once the trials are complete. Some initial guidance may be obtained by preclinical studies, especially in animal models that are close to the clinical situation either in the disease and/or in the organ size and geometry. Usually, data from early-phase trials are used to perform a statistical power calculation: that is, data from phase I are used to inform the likely number of patients needed in phase II, from which the results are then used to perform an evidence-based estimate of the numbers needed in phase III. There are established statistical methods to do these calculations, but guidance can also be obtained from published clinical trials of competing technologies for similar clinical applications. Significant effort can be saved by consulting with professional biostatisticians experienced in clinical trial design and analysis, especially if they are also experienced in medical device trials. It is also advisable to consult with more than one clinical end-user, to understand and address potential biases: this input should include consultation with clinicians (physicians, nurses, and other clinical support staff) across the full spectrum of intended clinical environments for the product and spanning the relevant range of expertise. One caution is that community hospitals, local clinics, and individual practitioners who may determine the ultimate market for the technology can have very different perspectives from the "thought leaders" at large academic centers who are often involved in the R\&D phases.

\subsection{Commercialization and Technology}

There are, of course, many obstacles that comprise the so-called valley(s) of death ${ }^{3}$ that must be crossed to get new technologies into the hands of clinicians. Here, we will only give a few examples where the commercial and R\&D/clinical domains intersect directly. The first is what the technology claims to do, from a regulatory perspective. This could be as simple as "... this optical imaging device characterizes tissue..." without claiming any specific application, disease, or clinical impact. At the other extreme would be, e.g., "... this near-infrared fiberoptic fluorescence spectroscopy device and algorithm reduces the rate of second surgeries in breast cancer lumpectomy procedures by at least $15 \%$...." Clearly, in the first case, the barriers to obtaining regulatory approval, assuming that all safety requirements are met, are much lower than in the second case, which 
would likely require a multicenter trial with a substantial number of patients. On the other hand, how large is the market for the first technology and who would reimburse its use in the clinic? Nevertheless, it may be advantageous to use the first approach as a stepping stone to the second. Moreover, the choice of an appropriate predicate can help facilitate initial regulatory approval.

In terms of consumables, many OSI technologies are comprised of fixed hardware (e.g., a spectroscopy system or multiwavelength imager) and operate in "label-free" mode, i.e., are based on the intrinsic optical properties of the biosample. ${ }^{62}$ This includes both linear optical techniques and emerging nonlinearbased OSI approaches. However, potential consumables, i.e., components that are used only once or a small number of times, may be required to achieve adequate sensitivity and/or specificity. These include fiberoptic probes/catheters for in vivo spectroscopy, cuvettes for lab-on-a-chip devices used in a fixed read-out instrument, as well as exogenous "reporters," such as fluorophores, nanoparticles, and photosensitizers. The relative advantages and limitations of label-free versus exogenous agents are many and complex and have been the subject of ongoing debates in the biomedical optics community. ${ }^{62-64}$ From a commercial perspective, consumables are usually highly desirable since they represent a continuing revenue stream, but their use often makes the regulatory process much more challenging. This is especially the case if the exogenous agent is actually administered to the patient rather than being used as an ex vivo "stain" as for histopathology, since safety (usually requiring arms-length toxicology studies in multiple animal species including large animals such as dogs, and performed by certified companies in multiple animal species), biodistribution, pharmacokinetics, etc. must all be determined and the materials must be produced under good-manufacturing-practice conditions.

\section{Other Challenges in Clinical Translation}

\subsection{Safety}

The most important aspect of any new technology intended for clinical use is that it should do no harm, either to the patient or personnel using it. This applies also in the clinical trials. The potential harm may be direct, as in damage caused by a laser beam in the OSI system or toxic effects of optically active exogenous compounds, or indirect as in causing changes to tissue samples such that histopathology readout and hence clinical diagnosis is compromised. Typically, in clinical trials the OSI information should not be used to alter patient care until after it has been demonstrated that the information is reliable: e.g., a new optical biopsy device should not be used to guide the surgeon to remove more tumor until there is an acceptable level of confidence that the technique does indeed reliably detect malignant tissue, based on prior "passive" (i.e., noninterventional) trials. It is particularly important to confirm which of false-positive or false-negative findings in individual patients, in clinical trials and beyond, could place the patient at higher risk: leading to over treatment or under treatment, respectively.

Although most diagnostic OSI applications use low optical power and energy (densities) that are within the usual exposure standards, these standards are based primarily on data from skin and eye exposure. The applicability of these to exposure of internal organs is not well determined, and UV or multiphoton exposure may be of particular concern because of potential mutagenesis. ${ }^{64}$ Published clinical studies of endoscopic techniques can provide useful insight and baseline information on this issue, at least for some wavelength ranges and optical pulse widths.

\subsection{Preclinical Models}

Preclinical R\&D to optimize and test OSI systems for ultimate use in patients often involves the use of cellular and/or animal models of disease. Especially for in vivo clinical applications, there may be a requirement for safety, biodistribution, and toxicity and effectiveness testing in animals. The basic requirement is to replicate as closely as possible the intended human use with respect to the specific OSI technology and the intended clinical utility claims. This last point is often overlooked. For example, there are many publications on OSI for early cancer detection using transplanted tumors in rodents but there are several typical shortcomings of these models: unlike human tumors, they are not spontaneous; the tumors are often placed accessibly under the skin or in muscle, whereas the detection in patients is on a background of the organ of origin; the tumors are often not of human origin and so may not have the true biochemical or structural characteristics; and the models do not represent early or precancerous lesions that are often the clinical target, at least for diagnostics. Thus, for example, a sensitivity and specificity above $90 \%$ for detecting transplanted mouse tumors relative to mouse muscle may have little relevance to detecting premalignant areas in the esophagus of patients. However, such experiments may be informative with respect to the technical performance of the OSI system, and poor performance in these less challenging situations can provide an early indicator of whether a technology is worth pursuing. If for no other reasons than expense and time, it is always worth asking whether it is better to use an animal model in vivo or to utilize ex vivo human tissues (e.g., fresh biopsy or from a tissue bank or archive) or to begin directly with patients, assuming that safety has been demonstrated and there is reason to believe that the technique may be effective.

\subsection{Gold Standards}

In validating in vivo clinical OSI techniques the standard of comparison is often the current clinical technique, either biochemical assay of a body fluid, cytology of cell samples, or histopathology of biopsied or resected tissue taken concurrently with the optical spectra or images. Indeed, this may be an absolute requirement for institutional ethical approval of clinical trials and ultimate regulatory approval. Such established methods are considered the gold standard. However, it should be realized that these may not be as reliable as the name implies, especially for conditions, such as early cancer detection, for which the sensitivity and specificity may be rather poor and the agreement between pathologists can be low. A further problem is that, for example, in "optical biopsy" the measurement may not be made in exactly the same location as the biopsy is taken due to unavoidable tissue motion or operator error. Hence, the optical measurement may be misinterpreted as false-positive or false-negative relative to the biopsy. Further, even if the tissue location is identical, the highest grade of tumor (which is what the pathologist usually reports) may represent only a small fraction of the sampling volume of the optical measurement, thereby underestimating the sensitivity of the optical method. This may be partially circumvented by requiring a more detailed 
histopathological analysis and report (e.g., estimating the fraction of the sample that shows disease) but at added cost and time. Accounting for tissue heterogeneity relative to biopsy sampling volume is a challenge, due to the effect of tissue optical properties on sampling volume and the difficulty in providing weighted volumetric histopathology results.

A further issue in using the current gold standards as the reference is that they are intrinsically limited in their ability to stratify patients, i.e., they can provide a diagnostic of disease $X$ with grade $Y$, but there are subsets of those patients that do not, for example, respond equally well to the therapy. Developing OSI to its full potential will then rely on validation against new approaches, either in the form of genetic testing or more specific molecular-pathology techniques and/or new bio data processing approaches, such as the application of artificial intelligence (machine learning and deep learning). The overall objective is to establish statistically rigorous models to improve patient stratification and predict outcome.

\subsection{Technology Standardization}

Unlike more established clinical radiological imaging technologies (e.g., MRI, CT, and nuclear imaging), there is currently a lack of well-established and widely accepted methods of standardizing and calibrating OSI techniques, i.e., there are few if any standard operating practices (SOP). For example, Raman spectroscopy for in vivo detection of cervical cancers has been carried out by various groups using different instruments and algorithms in diverse populations to show improved classification efficiency, but it would be more fruitful in further studies to establish SOPs for clinical implementation. ${ }^{65}$ In part, this is because the techniques and technologies (devices, software, and photoactive compounds) are very diverse, as is the industry base, which has many early-stage companies and only a few large multinational companies and institutions. The professional societies for institution- and industry-based researchers have not typically had strong participation in clinical translation, while conversely those of the clinical users have had little involvement in OSI technology development and promotion. This is beginning to change, as evidenced by recent initiatives by government agencies and professional bodies, both in Europe and the USA, to develop guidelines for clinical biomedical optics: for example, in the clinical uses of fluorescence-guided surgery and the minimum performance levels of the corresponding technologies. ${ }^{8}$

\subsection{Multimodality Optical Spectroscopy and Imaging}

Finally, it is most often the case that a single OSI technology does not adequately address the unmet clinical need, frequently because its sensitivity and/or specificity are too low for clinical adoption. There is then the option to combine the technique with one or more other techniques, which may be optical or nonoptical and either established or also under development, to provide the needed complementary information or therapeutic capability. For example, for the unmet clinical need of identifying dysplastic (premalignant) tissues in the esophagus of patients with chronic inflammatory disease (Barrett's esophagus), many OSI methods have been investigated: ${ }^{66-69}$ some have good sensitivity but poor specificity (e.g., tissue autofluorescence), while others have both high sensitivity and specificity but are slower and provide only point sampling (e.g., Raman spectroscopy). Subsurface imaging to stage the disease is also of high added value, since it determines the treatment options in cases where dysplasia is found: potentially, OCT or photoacoustic imaging may have utility for this purpose. Combining some of these techniques has been shown to be more effective than any one technique alone, as has been the case for various OSI tools and applications. ${ }^{70,71}$ Of course, a major challenge then is how to combine the techniques into a single device. "Bottom-up" integration is preferred over simply running the techniques in parallel, but can pose considerable technical difficulties, for example, if different optical fiber characteristics are needed for the different methods. A second challenge in multimodality OSI, from a commercialization perspective, is that the IP ownership is likely to be distributed between multiple individuals, institutions, or companies, so that combining these into a common enterprise may not be possible. Moreover, clinical adoption of technologies is often incremental and most readily achieved by creating techniques that complement the current standard of care. Therefore, any opportunity to create a multimodal system combining an optical technique with an alreadyadopted modality (e.g., MRI, CT, etc.) may be easier to translate to the clinic than combining two unproven technologies. Ideally, new technologies should provide valuable information to clinical users while conforming to standard practice with minimal disruption to the clinical workflow.

\section{Conclusions}

OSI, and the field of biomedical optics (biophotonics) in general, have seen rapid growth in the last few decades, enabled by the many advances in optical sciences and technologies and the convergence with complementary fields, such as molecular biology, chemistry, nanotechnology, robotics, imaging, computation/data management, machine and deep learning/artificial intelligence, and computer vision. Simultaneously, major trends in clinical medicine have provided new opportunities and challenges for both high- and low-tech approaches. These include the need to address major drivers such as aging populations and increasing chronic and degenerative diseases in the developed world, the global re-emergence of infectious diseases, personalized medicine, and low-cost point-of-care health care delivery. OSI approaches are well positioned to make significant contributions across all of these, through both basic enabling R\&D and clinical translation. While, as discussed in this paper, the latter presents many and diverse hurdles, the exceptional intrinsic and practical capabilities of OSI mean that we should see its increased utilization across a range of clinical settings. Many of the challenges identified here as being faced by OSI are also relevant to various other nonoptical technologies. Finally, innovation in any field, including OSI, is ultimately dependent on scientific and technological creativity, a spontaneous activity. However, translating this into clinical practice is a more managed process and this paper, as well as other recent publications ${ }^{1-3}$ have attempted to layout some of the structure for this.

\section{Disclosures}

F.L. is cofounder and chief technical officer (CTO) of and has ownership interest in ODS Medical Inc. M.J. is employed by DoseOptics LLC.

\section{Acknowledgments}

Support of the Terry Fox Research Institute, the National Institutes of Health (US) and the Princess Margaret Foundation is acknowledged (BCW). 


\section{References}

1. L. Marcu et al., "Biophotonics: the big picture," J. Biomed. Opt. 23(2), 021103 (2017).

2. D. M. Beswick et al., "Biomedical device innovation methodology: applications in biophotonics," J. Biomed. Opt. 23(2), 021102 (2017).

3. J. Popp et al., "Challenges in translation: models to promote translation," J. Biomed. Opt. 23(2), 021101 (2017).

4. S. Takehana, M. Kaneko, and H. Mizuno, "Endoscopic diagnostic system using autofluorescence," Diagn. Ther. Endosc. 5(2), 59-63 (1999).

5. A. Garcia-Uribe et al., "In vivo diagnosis of melanoma and nonmelanoma skin cancer using oblique incidence diffuse reflectance spectrometry," Cancer Res. 72(11), 2738-2745 (2012).

6. M. Jermyn et al., "Intraoperative brain cancer detection with Raman spectroscopy in humans," Sci. Transl. Med. 7(274), 274 ra19 (2015).

7. W. Stummer et al., "Fluorescence-guided surgery with 5-aminolevulinic acid for resection of malignant glioma: a randomised controlled multicentre phase III trial," Lancet Oncol. 7(5), 392-401 (2006).

8. S. B. Mondal et al., "Real-time fluorescence image-guided oncologic surgery," Adv. Cancer Res. 124, 171-211 (2014).

9. B.-W. Xie et al., "Dual-wavelength imaging of tumor progression by activatable and targeting near-infrared fluorescent probes in a bioluminescent breast cancer model," PLoS One 7(2), e31875 (2012).

10. E. L. Kaijzel, G. van der Pluijm, and C. W. G. M. Löwik, "Whole-body optical imaging in animal models to assess cancer development and progression," Clin. Cancer Res. 13(12), 3490-3497 (2007).

11. C. G. Hadjipanayis et al., "Current and future clinical applications for optical imaging of cancer: from intraoperative surgical guidance to cancer screening," Semin. Oncol. 38(1), 109-118 (2011).

12. S. Feng et al., "Nasopharyngeal cancer detection based on blood plasma surface-enhanced Raman spectroscopy and multivariate analysis," Biosens. Bioelectron. 25(11), 2414-2419 (2010).

13. C. Hirche et al., "ICG fluorescence-guided sentinel node biopsy for axillary nodal staging in breast cancer," Breast Cancer Res. Treat. 121(2), 373-378 (2010).

14. F. T. Nguyen et al., "Intraoperative evaluation of breast tumor margins with optical coherence tomography," Cancer Res. 69(22), 8790-8796 (2009).

15. A. S. Haka et al., "In vivo margin assessment during partial mastectomy breast surgery using Raman spectroscopy," Cancer Res. 66(6), 33173322 (2006).

16. D. E. J. G. J. Dolmans, D. Fukumura, and R. K. Jain, "Photodynamic therapy for cancer," Nat. Rev. Cancer 3(5), 380-387 (2003).

17. D. van Straten et al., "Oncologic photodynamic therapy: basic principles, current clinical status and future directions," Cancers 9(2), 19 (2017).

18. S. H. Halani and D. C. Adamson, "Clinical utility of 5-aminolevulinic acid $\mathrm{HCl}$ to better visualize and more completely remove gliomas," OncoTargets Ther. 9, 5629-5642 (2016).

19. N. Sanai et al., "Intraoperative confocal microscopy in the visualization of 5-aminolevulinic acid fluorescence in low-grade gliomas," $J$. Neurosurg. 115(4), 740-748 (2011).

20. C. Krafft et al., "Developments in spontaneous and coherent Raman scattering microscopic imaging for biomedical applications," Chem. Soc. Rev. 45(7), 1819-1849 (2016).

21. S. L. Jacques, "Optical properties of biological tissues: a review," Phys. Med. Biol. 58(11), R37-R61 (2013).

22. B. Das et al., "Alzheimer mouse brain tissue measured by time resolved fluorescence spectroscopy using single- and multi-photon excitation of label free native molecules," J. Biophotonics 11, e201600318 (2017).

23. R. Genthial et al., "Label-free imaging of bone multiscale porosity and interfaces using third-harmonic generation microscopy," Sci. Rep. 7(1), 3419 (2017)

24. K. Reiser, P. Stoller, and A. Knoesen, "Three-dimensional geometry of collagenous tissues by second harmonic polarimetry," Sci. Rep. 7(1), 2642 (2017)

25. A. Khmaladze et al., "Hyperspectral imaging and characterization of live cells by broadband coherent anti-stokes Raman scattering (CARS) microscopy with singular value decomposition (SVD) analysis," Appl. Spectrosc. 68(10), 1116-1122 (2014).

26. C.-S. Liao et al., "Spectrometer-free vibrational imaging by retrieving stimulated Raman signal from highly scattered photons," Sci. Adv. 1(9), e1500738 (2015).
27. C. Kut et al., "Detection of human brain cancer infiltration ex vivo and in vivo using quantitative optical coherence tomography," Sci. Transl. Med. 7(292), 292ra100 (2015)

28. F. A. South et al., "Differentiation of ex vivo human breast tissue using polarization-sensitive optical coherence tomography," Biomed. Opt. Express 5(10), 3417-3426 (2014).

29. A. Beljebbar et al., "Ex vivo and in vivo diagnosis of C6 glioblastoma development by Raman spectroscopy coupled to a microprobe," Anal. Bioanal. Chem. 398(1), 477-487 (2010).

30. M. Jermyn et al., "A review of Raman spectroscopy advances with an emphasis on clinical translation challenges in oncology," Phys. Med. Biol. 61(23), R370-R400 (2016).

31. L. A. Austin, S. Osseiran, and C. L. Evans, "Raman technologies in cancer diagnostics," Analyst 141(2), 476-503 (2016).

32. I. Pence and A. Mahadevan-Jansen, "Clinical instrumentation and applications of Raman spectroscopy," Chem. Soc. Rev. 45(7), 1958-1979 (2016).

33. G. Hong, A. L. Antaris, and H. Dai, "Near-infrared fluorophores for biomedical imaging," Nat. Biomed. Eng. 1(1), 0010 (2017).

34. R. Singh et al., "White light endoscopy, narrow band imaging and chromoendoscopy with magnification in diagnosing colorectal neoplasia," World J. Gastrointest. Endosc. 1(1), 45-50 (2009).

35. H. Vaziri and J. C. Anderson, "White light endoscopy versus chromoendoscopy for the detection of dysplasia during inflammatory bowel disease surveillance with colonoscopy," Gastroenterology 149(6), 16301632 (2015)

36. M. Yung, M. A. Klufas, and D. Sarraf, "Clinical applications of fundus autofluorescence in retinal disease," Int. J. Retina Vitreous $\mathbf{2}$, 12 (2016).

37. M. Adhi and J. S. Duker, "Optical coherence tomography—current and future applications," Curr. Opin. Ophthalmol. 24(3), 213-221 (2013).

38. J. A. Dawson et al., "Pulse oximetry for monitoring infants in the delivery room: a review," Arch. Dis. Child. 92(1), F4-F7 (2007).

39. P. Z. McVeigh et al., "High-resolution angioscopic imaging during endovascular neurosurgery," Neurosurgery 75(2), 171-180 (2014).

40. M. Jermyn et al., "Raman spectroscopy detects distant invasive brain cancer cells centimeters beyond MRI capability in humans," Biomed. Opt. Express 7(12), 5129-5137 (2016).

41. C. T. Lam et al., "Design of a novel low cost point of care tampon (POCkeT) colposcope for use in resource limited settings," PLoS One 10(9), e0135869 (2015).

42. K. C. Blanco et al., "Clinical photodynamic therapy review and the Brazilian experience," J. Tumor 4(2), 386-392 (2016).

43. A. L. Whitehead et al., "Estimating the sample size for a pilot randomised trial to minimise the overall trial sample size for the external pilot and main trial for a continuous outcome variable," Stat. Methods Med. Res. 25(3), 1057-1073 (2016).

44. S. D. Halpern, J. H. T. Karlawish, and J. A. Berlin, "The continuing unethical conduct of underpowered clinical trials," JAMA 288(3), 358-362 (2002).

45. P. Lee et al., "Color fluorescence ratio for detection of bronchial dysplasia and carcinoma in situ," Clin. Cancer Res. 15(14), 4700-4705 (2009).

46. K. Nakanishi et al., "Color auto-fluorescence from cancer lesions: improved detection of central type lung cancer," Lung Cancer 58(2), 214-219 (2007)

47. T. Mang et al., "Autofluorescence and Photofrin-induced fluorescence imaging and spectroscopy in an animal model of oral cancer," Photodiagn. Photodyn. Ther. 3(3), 168-176 (2006).

48. Q. R. J. G. Tummers et al., "Real-time intraoperative detection of breast cancer using near-infrared fluorescence imaging and methylene blue," Eur. J. Surg. Oncol. 40(7), 850-858 (2014).

49. C. Zhu et al., "Diagnosis of breast cancer using fluorescence and diffuse reflectance spectroscopy: a Monte-Carlo-model-based approach," $J$. Biomed. Opt. 13(3), 034015 (2008).

50. Z. Volynskaya et al., "Diagnosing breast cancer using diffuse reflectance spectroscopy and intrinsic fluorescence spectroscopy," $J$. Biomed. Opt. 13(2), 024012 (2008).

51. F. Schnabel et al., "A randomized prospective study of lumpectomy margin assessment with use of MarginProbe in patients with nonpalpable breast malignancies," Ann. Surg. Oncol. 21(5), 1589_ 1595 (2014). 
52. T. C. Kennedy, S. Lam, and F. R. Hirsch, "Review of recent advances in fluorescence bronchoscopy in early localization of central airway lung cancer," Oncologist 6(3), 257-262 (2001)

53. P. A. Valdés et al., "Quantitative, spectrally-resolved intraoperative fluorescence imaging," Sci. Rep. 2, 798 (2012).

54. R. S. DaCosta et al., "Point-of-care autofluorescence imaging for realtime sampling and treatment guidance of bioburden in chronic wounds: first-in-human results," PLoS One 10(3), e0116623 (2015).

55. D. Cozzolino et al., "Multivariate data analysis applied to spectroscopy: potential application to juice and fruit quality," Food Res. Int. 44(7), 1888-1896 (2011).

56. T. Bocklitz et al., "How to pre-process Raman spectra for reliable and stable models?" Anal. Chim. Acta 704(1-2), 47-56 (2011).

57. A. Abdolmanafi et al., "Deep feature learning for automatic tissue classification of coronary artery using optical coherence tomography," Biomed. Opt. Express 8(2), 1203-1220 (2017).

58. E. Bauer and R. Kohavi, "An empirical comparison of voting classification algorithms: bagging, boosting, and variants," Mach. Learn. 36(1-2), 105-139 (1999).

59. M. A. Hall and L. A. Smith, "Feature selection for machine learning. comparing a correlation-based filter approach to the wrapper," in Proc. of the Twelfth Int. Florida Artificial Intelligence Research Society Conf. pp. 235-239, AAAI Press (1999).

60. C. S. Lee et al., "Deep-learning based, automated segmentation of macular edema in optical coherence tomography," Biomed. Opt. Express 8(7), 3440-3448 (2017).

61. P. A. Valdés et al., "Combined fluorescence and reflectance spectroscopy for in vivo quantification of cancer biomarkers in lowand high-grade glioma surgery," J. Biomed. Opt. 16(11), 116007 (2011).

62. S. A. Boppart et al., "Label-free optical imaging technologies for rapid translation and use during intraoperative surgical and tumor margin assessment," J. Biomed. Opt. 23(2), 021104 (2017)

63. B. P. Joshi and T. D. Wang, "Exogenous molecular probes for targeted imaging in cancer: focus on multi-modal imaging," Cancers 2(2), 12511287 (2010)

64. M. C. Pierce, D. J. Javier, and R. Richards-Kortum, "Optical contras agents and imaging systems for detection and diagnosis of cancer," Int. J. Cancer 123(9), 1979-1990 (2008).

65. S. Rubina and C. M. Krishna, "Raman spectroscopy in cervical cancers: an update," J. Cancer Res. Ther. 11(1), 10-17 (2015).
66. L. B. Lovat et al., "Elastic scattering spectroscopy accurately detects high grade dysplasia and cancer in Barrett's oesophagus," Gut 55(8), 1078-1083 (2006)

67. K. Kong et al., "Raman spectroscopy for medical diagnostics-from in-vitro biofluid assays to in-vivo cancer detection," $A d v$. Drug Delivery Rev. 89, 121-134 (2015).

68. E. Rodriguez-Diaz et al., "Elastic scattering spectroscopy as an optical marker of inflammatory bowel disease activity and subtypes," Inflamm. Bowel Dis. 20(6), 1029-1036 (2014).

69. L. Qiu et al., "Multispectral scanning during endoscopy guides biopsy of dysplasia in Barrett's esophagus," Nat. Med. 16(5), 603-606 (2010).

70. C. A. Lieber et al., "Multimodal near infrared spectral imaging as an exploratory tool for dysplastic esophageal lesion identification," Opt. Express 14(6), 2211-2219 (2006).

71. M. Jermyn et al., "Highly accurate detection of cancer in situ with intraoperative, label-free, multimodal optical spectroscopy," Cancer Res. 77(14), 3942-3950 (2017)

Brian C. Wilson is a professor of medical biophysics at Princess Margaret Cancer Centre/University of Toronto, where he directs a multidisciplinary program in translational biophotonics. This spans optical diagnostics, therapeutic guidance and light-based therapeutics and, increasingly, enabled by optically active nanotechnologies.

Michael Jermyn has expertise in computational solutions for biomedical applications, with experience in machine learning, computer vision, cancer imaging, software development, and biomedical optics. $\mathrm{He}$ received his PhD from the Thayer School of Engineering, Dartmouth College, and completed a postdoctoral fellowship at the Montreal Neurological Institute, McGill University.

Frederic Leblond, $\mathrm{PhD}$, is an associate professor at the Department of Engineering Physics at Polytechnique Montreal, where he heads the Optical Radiology Laboratory (LRO). He is also adjunct professor at Dartmouth College and a researcher at the Centre for Research Hospital of the University of Montreal (CHUM Research Centre). His research program investigates the use of optical imaging and spectroscopy techniques for diagnostics and surgical guidance, with a focus on clinical translation. 